\title{
Novel Compact CPW LowPass Filter Integrating Periodic Triangle DGS Cells
}

\author{
${ }^{1}$ Elmahjouby Sghir, ${ }^{1}$ Ahmed Errkik, ${ }^{1}$ Jamal Zbitou, ${ }^{1}$ A.Tajmouati, ${ }^{1}$ Larbi El Abdellaoui, \\ ${ }^{2}$ Mohamed Latrach \\ ${ }^{2}$ LMEET Laboratory FST of Settat Hassan 1st University, Settat, Morocco \\ ${ }^{2}$ Microwave group ESEO, Angers, France \\ elmahjouby.sghir@gmail.com
}

\begin{abstract}
In this paper, we introduce a new periodic structure for CPW of a low pass filter based on the DGS technique with triangle slot cell forme. The proposed structure is a minuature low pass filter that exhibits low insertion loss in the passband, simple fabrication, and easy to integrate with microwave planar circuits. A Lowpass filter (LPF) has been designed with flat response at a selected frequency by cascading three DGS cells.
\end{abstract}

Keywords-cpw lowpass filter; triangle DGS; periodic cells; wide passband;

\section{Introduction}

Periodic structures of various types in coplanar wave guide (CPW) technologies has a potentially high volume demands in microwave and millimetre-wave domains to their filtering properties or inhibition of signal propagation in certain frequency bands. Also, Defected Ground Structures (DGS) have been developed to improve response and characteristics of microwave components. So when both of them combined in the same structure will give excellent performance in terms of response as bandwidth, quality factor and the parasite rejection, also the level of integration as the size, the development and integration of other components. The microwave circuits such as filters design[1], antennas [2], phase shifters [3], and other are of much application domain of CPW with DGS structure.

All this attractive of CPW-DGS structures because of useful for fabrication, characterisation and manufacture of a small, low-cost and highly integrated transceiver. However, promising application of $\mathrm{CPW}$ in microwave integrated circuits (MICs), monolithic microwave integrated circuits (MMICs), and microelectromechanical systems (MEMS) devices[4, 5].

In transmission and reception channels of modern microwave systems, such a wireless and mobile communications, the harmonics and spurious responses are caused by the caractiristic response of some no-linear devices like oscillator. So, it is necessaire to suppress harmonics and spurious signals in the bandpass signal. For this purpose, a compact CPW Low-pass filter (CPW-LPF) using DGS periodics structure is a good condidate to improve passband performances. 
Elmahjouby Sghir, Ahmed Errkik, Jamal Zbitou, A.Tajmouati, Larbi El Abdellaoui, Mohamed Latrach. A Novel Compact CPW LowPass Filter Integrating Periodic Triangle DGS Cells. Transactions on Machine Learning and Artificial Intelligence, Vol 5 No4 August (2017); p: 457-462

In this paper, a novel compact low pass filter meeting these requirements (CPW, DGS, Periodic cells) with high performances: Harmonic-suppressed, sharp attenuation and wide stop-band.

\section{Initilal DGS LPF Design}

\subsection{CPW line based on one Triangle DGS}

The geometry of the proposed Low Pass filter using Triangle DGS filter is depicted in Figure 1. The design started with one conventional cell, the DGS configuration in this structure is an Isosceles triangle slot etched in the ground

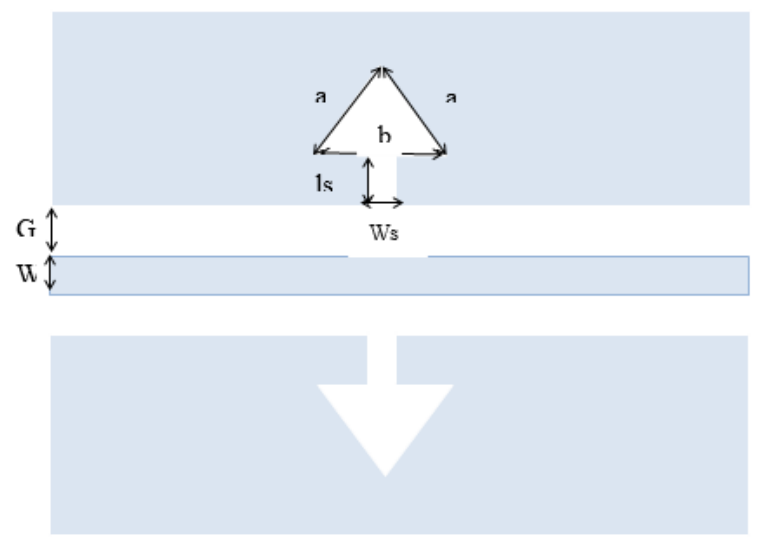

Figure. 1. CPW line with Triangle DGS structure

The coplanar waveguide line is designed to have a charactiristic impedance $Z 0=50 \Omega$, and built on standard 25-mil Duroid substrate with dielectric constant $\varepsilon r=10.2,[6]$. The central signal line width of $W=1.2 \mathrm{~mm}$, and the gap width is $G=0.42 \mathrm{~mm}$. Also the parameters of the triangle DGS are $a=1.1181 \mathrm{~mm}, b=1.0004$ $\mathrm{mm}$, and $\mathrm{Is}=\mathrm{ws}=0.42 \mathrm{~mm}$. The overall dimensions of the simulated device measures $20 \times 14.04 \mathrm{~mm} 2$. The parametric studies and simulation of the filter's electrical performances were performed using Momentum (an EM solver integred in ADS Agilent).

\subsection{Response of CPW DGS Triangle}

The Tringle DGS element etched in the ground plane has the effective capacitance (C) and inductance (L). The simulation insertion and return losses of the proposed topoligie filter is depicted in Figure 2.

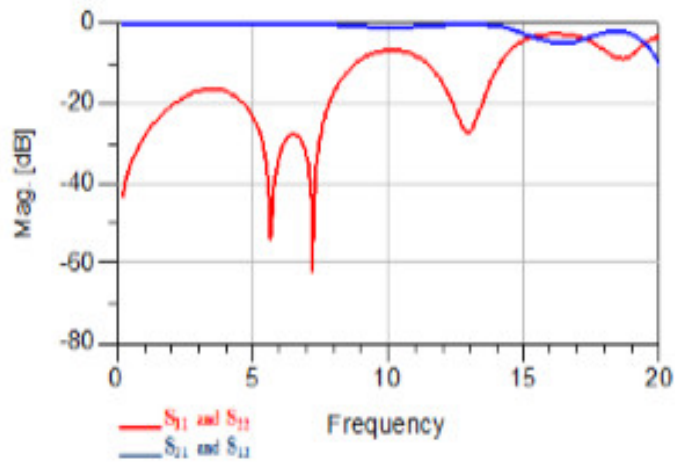

Figure. 2. Simulated S-parameters versus frequency for the CPW DGS Triangle structure. 
From the S-parameter responses, it is clear that the filter provides low pass band behavior, with large bandpass from 0.1 to $15 \mathrm{GHz}$, very low and flat insertion loss of $-0.5 \mathrm{~dB}$ and the minimum return loss of $10 \mathrm{~dB}$ in passband, but the suppression is inadequate because limit on the separation between the insertion loss and return loss in the stopband.

\section{Proposed Device of LPF with Periodic DGS Cells}

\subsection{CPW line based on Three Triangle DGS Cells}

In the research, augmentation of DGS elements number were used as a good solution to design a compact microwave filter, $[7,8,9]$. The performance of LPF with periodical DGSs was used here to have a much wider stopband compared with one DGS. Figure 3 depicts the schematic of the proposed structure with periodic cells

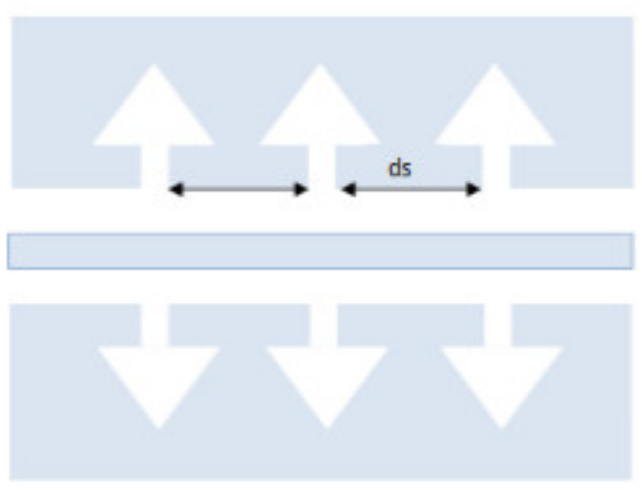

Figure. 3. The proposed device of periodics DGS Cells for LowPass filter

The parameters of the initial triangle DGS low pass filter are kept unchanged while two other symmetrical triangle DGS are introduced and separed with $\mathrm{ds}=2.9834 \mathrm{~mm}$ distance.

However, the insertion-loss and return-loss performance were inadequate. As a result, a LPF with three cells periodical triangle DGSs was designed in an attempt to improve the performance of S-parameters as shown in Fig.4.

Figure 4 shows the simulated S-parameters of the proposed device of low pass filter with three periodic Cells

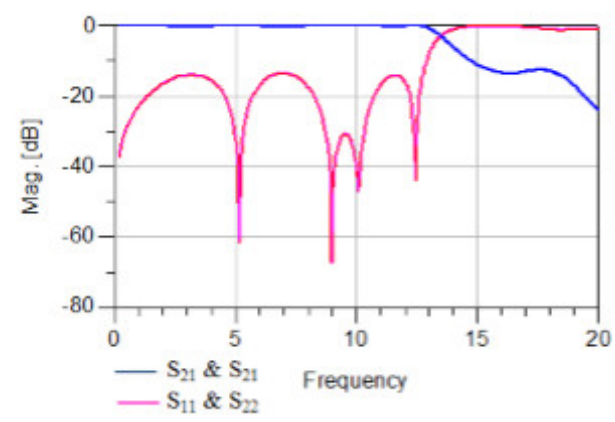

Figure. 4. Simulated S-parameter of the proposed periodic DGS cells device

As a result shown in Fig. 4, the cascaded LPF with the three periodic cells present a sharp roll-off with improved stop band and supression performance. The return loss in pass-band is greater than $15 \mathrm{~dB}$, with 
Elmahjouby Sghir, Ahmed Errkik, Jamal Zbitou, A.Tajmouati, Larbi El Abdellaoui, Mohamed Latrach. A Novel Compact CPW LowPass Filter Integrating Periodic Triangle DGS Cells. Transactions on Machine Learning and Artificial Intelligence, Vol 5 No4 August (2017); p: 457-462

no more than $0.3 \mathrm{~dB}$ ripple level in the pass-band, while the stop band rejection level is better than 10 $\mathrm{dB}$ with wide stopband of 13 to more than $20 \mathrm{GHz}$.

\subsection{Effect of additional periodic cells on filter responce.}

The simulation results of three Triangle DGS cells are shown in Figure 4. It is clearly shown in the figure that three-cell in the structure's ground is enough to suppress the spurious response in pass and stop bands and give out a wider stopband. Figures 5 and 6 demonstrates the effect of additional periodic cells on insertion and return loss.

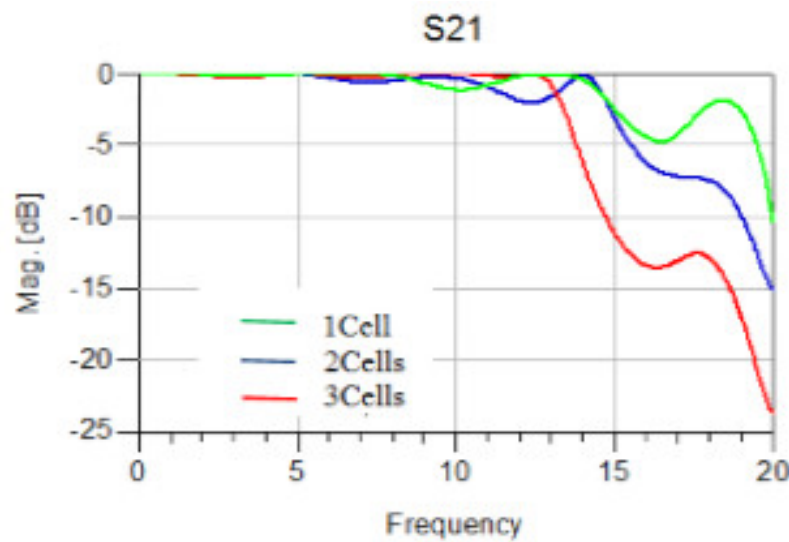

Figure. 5. Effect of additional periodic cells on filter insertion loss

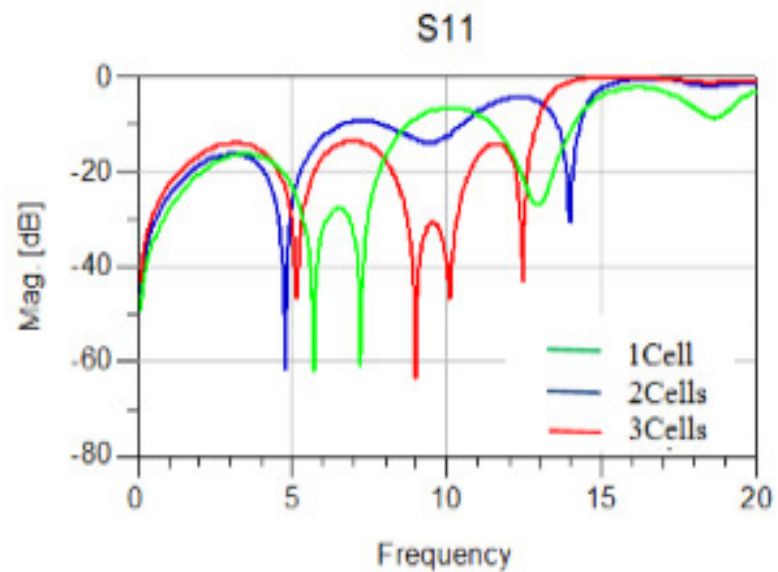

Figure. 6. Effect of additional periodic cells on filter return loss.

Figures 5 and 6 demonstrated that the augmentation of periodic tiangle DGS cells take a great effect on the filter response. There is a good acheiving of attenuation with no ripple level of insertion loss in the pass-band with just 3 cells. Also, the filter present spurious-free response and deep attenuation levels in the return loss $-60 \mathrm{~dB}$ in passband and rejection level better than $-20 \mathrm{~dB}$ in the stopband, when the number of DGS cells augmented. 


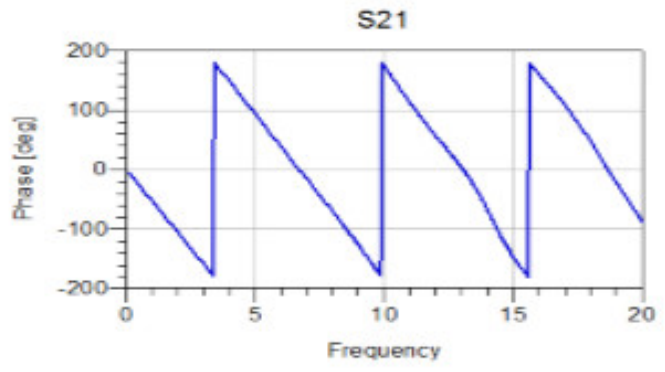

Figure. 7. Simulation Phase response of Lowpass Filter Designed.

Figure 7 shows the phase simulation of insertion loss (S21) for UWB bandpass between $0.1 \mathrm{GHz}$ and 20 $\mathrm{GHz}$ of designed lowpass filter that throughout the pass and stop bands is acceptably linear for UWB applications.

\section{Conclusion}

In this paper, a simple compact lowpass filter using triangle defected ground structure is proposed, developed and presented. With 3-Cells DGS series on the same substrate offers the advantages of miniature low-pass filter than a standard CPW transmission line, low insertion loss with no harmonics in the passband, high attenuation levels in the stopband and very easy fabrication, while the cutoff frequency can be adjusted by controlling the dimensions of the triangle DGS cell to obtained others response frequency for more wireless applications.

\section{ACKNOWLEDGMENT}

We thank Mr. Mhamed Latrach from ESEO Angers in France, for allowing us to use and to perform simulations by using electromagnetic solvers and measurement instruments

\section{REFERENCES}

[1] Hettak, K. and G. Delisle, “A new miniature uniplanar lowpass Filter using series resonators, " IEEE MTTS, Vol. 3, No. 3, 1193-1196, Jun. 7-12, 1998.

[2] Chang, I., and Lee, B. : "Design of defected ground structures for harmonic control of active microstrip antenna,". 2002 IEEE AP-S Int. Symp. Dig., 2002, pp. 852-855.

[3] Elamaran, B, Chio, I.M, Chen, L.Y, and Chiao, J.C, "A beamsteerer using reconfigurable PBG ground plane," 2000 IEEE MTT-S Int. Microwave Symp. Dig., 2000, pp. 835-838.

[4] S. Seki and H. Hasegawa, "Cross-tie slow-wave coplanar waveguide on semi-insulating GaAs substrate," Electron. Lett., vol. 17, no. 25, pp. 940-941, Dec. 1981.

[5] K. Wu and R. Vahldieck, "Hybrid-mode analysis of homogeneously and inhomogeneously doped low-loss slow-wave coplanar transmission lines," IEEE Trans. Microwave Theory Tech., vol. 39, no. 8, pp. 13481360, Aug. 1991. 
Elmahjouby Sghir, Ahmed Errkik, Jamal Zbitou, A.Tajmouati, Larbi El Abdellaoui, Mohamed Latrach. A Novel Compact CPW LowPass Filter Integrating Periodic Triangle DGS Cells. Transactions on Machine Learning and Artificial Intelligence, Vol 5 No4 August (2017); p: 457-462

[6] Jong-Sik Lim, Chul-Soo Kim, Young-Taek Lee, Dal Ahn, Sangwook Nam, "A Spiral-Shaped Defected Ground Structure for Coplanar Waveguide," IEEE Microwave and wireless conponents letters, vol. 12, no. 9, September 2002.

[7] J. Y. Wu, Y. H. Tseng and W. H. Tu, "Design of compact lowpass filter with ultra-wide stopband using thin slots," Progress In Electromagnetics Research C, Vol. 31, pp. 137-151, 2012.

[8] J. W. No and H. Y. Hwang, "A design of cascaded CPW low-pass filter with broad stopband," IEEE Microwave and Wireless Components Letters, Vol. 17, pp. 427-429, 2007.

[9] C. Han-Jan, H. Tsung-Hui, and C. Chin-Sheng et al., "A novel cross-shape DGS applied to design ultra-wide stopband low-pass filters," IEEE Microwave and Wireless Components Letters, Vol. 16, No. 5, 2006, pp. 252-254. 